\title{
Impact of anesthesia methods on perioperative systemic inflammation and long-term outcomes in patients undergoing surgery for hepatocellular carcinoma: a propensity score-matched analysis
}

\author{
Hao Zhang ${ }^{1 \#}$, Kefang Guo ${ }^{1 \#}$, Xingfeng Sun ${ }^{1 \#}$, Yi Liu ${ }^{4,5}$, Ziwen Zhong ${ }^{1}$, Wenchang Zhou ${ }^{1}$, Weian Zeng ${ }^{6}$, \\ Lu Wang ${ }^{7}$, Juan P. Cata ${ }^{2,3}$, Wankun Chen ${ }^{1}$, Changhong Miao ${ }^{1}$ \\ ${ }^{1}$ Department of Anesthesiology, Zhongshan Hospital, Fudan University, Shanghai, China; ${ }^{2}$ Department of Anesthesiology and Perioperative \\ Medicine, The University of Texas MD Anderson Cancer Centre, Houston, USA; ${ }^{3}$ Anaesthesiology and Surgical Oncology Research Group, \\ Houston, USA; ${ }^{4}$ Department of Anesthesiology, Fudan University Shanghai Cancer Centre, Shanghai, China; ${ }^{5}$ Department of Oncology, Shanghai \\ Medical College, Fudan University, Shanghai, China; ${ }^{6}$ Department of Anesthesiology, Sun Yat-sen University Cancer Centre, State Key Laboratory \\ of Oncology in South China, Collaborative Innovation Centre for Cancer Medicine, Guangzhou, China; ${ }^{7}$ Department of Liver Surgery, Shanghai \\ Cancer Center, Shanghai Medical College, Fudan University, Shanghai, China
}

Contributions: (I) Conception and design: H Zhang, W Chen, JP Cata; (II) Administrative support: X Sun, K Guo, Y Liu, Z Zhong; (III) Provision of study materials or patients: L Wang, W Zeng, W Zhou; (IV) Collection and assembly of data: X Sun, K Guo, Y Liu, Z Zhong; (V) Data analysis and interpretation: H Zhang, W Chen, JP Cata, C Miao; (VI) Manuscript writing: All authors; (VII) Final approval of manuscript: All authors.

"These authors contributed equally to this work.

Correspondence to: Changhong Miao, MD, PhD. Department of Anesthesiology, Zhongshan Hospital, Fudan University, 180\# Feng-Lin Road, Shanghai 200032, China. Email: miaochangh@163.com; Wankun Chen, MD, PhD. Department of Anesthesiology, Zhongshan Hospital, Fudan University, 180\# Feng-Lin Road, Shanghai 200032, China. Email: chenwank@163.com; Juan P. Cata, MD. Department of Anesthesiology and Perioperative Medicine, The University of Texas MD Anderson Cancer Centre, Unit-409, 1515 Holcombe Blvd, Houston, Texas 77030-4009, USA. Email: jcata@mdanderson.org.

Background: Recent studies have shown regional anesthesia might improve the survival of cancer patients. We hypothesized that general-epidural anesthesia (GEA) was associated with longer survival than general anesthesia (GA) in patients undergoing hepatocellular carcinoma (HCC) resections.

Methods: A retrospective study included patients who received curative resection for HCC between January 2014 to December 2017. Patients were grouped in GEA vs. GA. After propensity score matching, perioperative inflammatory scores were calculated. Grade of postoperative complications, length of stay (LOS), dosage of sufentanil used and times of patients requiring rescue analgesia in both groups were compared for intraoperative and postoperative parameter. Survival curves were constructed from the date of surgery to death, univariable and multivariable Cox regression models were used to compare hazard ratios for death.

Results: A total of 772 patients were included in the study. With 386 patients in GA group and 386 patients in GEA group. After propensity score matching, the demographic and baseline biomarkers in the two groups were similar. Patients in GEA group showed significantly lower inflammatory scores. Grade of postoperative complications, LOS, opioid use, and times of patients requiring rescue analgesia was significantly lower in the GEA group. The overall survival (OS) and disease-free survival (DFS) rate was significantly lower in the GA group $(54.2 \%$ vs. $62.3 \%, 41.2 \%$ vs. $52.5 \%, \mathrm{P}<0.001)$. The multivariate analysis indicated the GA was associated with shorter OS (HR: 1.28, 95\% CI: 1.07-2.02, P<0.001) and DFS (HR: 1.06, 95\% CI: $1.03-1.71$, $\mathrm{P}<0.001)$.

Conclusions: GA combined with epidural anesthesia is associated with lower levels of inflammation, and longer survival in patients undergoing hepatectomy. 
Keywords: Anesthesia methods; hepatocellular carcinoma; perioperative systemic inflammation; long-term outcomes

Submitted May 24, 2020. Accepted for publication Sep 25, 2020.

doi: $10.21037 / \mathrm{atm}-20-3704$

View this article at: http://dx.doi.org/10.21037/atm-20-3704

\section{Introduction}

Worldwide, the incidence and mortality of liver cancer rank sixth and fourth, respectively, among malignant tumors (1). China is a region with a high incidence of liver cancer, and more than half of the new cases of liver cancer in the world every year occur in China (1-3). Surgical resection is still the preferred method for liver cancer treatment (4), but surgical trauma can lead to a series of systemic metabolic, inflammatory, and neuroendocrine responses, which in turn suppress the immune surveillance function of patients promoting postoperative cancer progression $(5,6)$. Recent study has shown that in addition to surgical trauma, a variety of perioperative factors can affect the antitumor immune function and are associated with cancer recurrence (7). Among these factors, increased attention has been paid to the impact of the choice of anesthesia methods on the perioperative anti-tumor immune function and the oncologic long-term prognosis (8-11).

Some inflammatory and immune responses related indicators, such as the neutrophil-to-lymphocyte ratio (NLR), the lymphocyte-to-monocyte ratio (LMR) and the systemic immune-inflammation index (SII), have been proposed as prognostic markers for malignant tumors $(12,13)$. A sub-analysis from an ongoing randomized controlled trial shows that regional analgesia in combination with propofol during breast cancer surgery attenuates the inflammatory response as measured by NLR (14). Another sub-analysis of patients undergoing hepatic resections for non-primary liver cancers demonstrates that epidural anesthesia strongly modulates the perioperative inflammatory response (15). However, it is unclear whether general anesthesia (GA) alone and GA combined with a thoracic epidural block [general-epidural anesthesia (GEA)] can affect those markers and translate in improved oncologic outcomes in patients undergoing liver cancer surgery. We hypothesize that the use of GEA (versus GA) is an independent predictor of longer survival in patients undergoing partial hepatectomy. Therefore, we conducted a retrospective study to evaluate the effect of different anesthetic and analgesic methods on perioperative inflammatory indicators, postoperative metastasis and recurrence and long-term prognosis in patients undergoing partial hepatectomy for primary liver cancers.

We present the following article in accordance with the STROBE reporting checklist (available at http://dx.doi. org/10.21037/atm-20-3704).

\section{Methods}

The study was conducted in accordance with the Declaration of Helsinki (as revised in 2013). This study was approved by the Ethics Committee of Fudan University Shanghai Cancer Center (FUSCC), China (No. 202003011-3). All patients were consent the data for research use when receive treatment. From January 2014 to December 2017, patients receiving selective curative resection for hepatocellular carcinoma at FUSCC were enrolled in this retrospective cohort. The inclusion criteria were as follows: postoperative pathology shows hepatocellular carcinoma, diagnosis of primary liver cancer stage I-III according to the eighth edition of American Joint Committee on Cancer (AJCC) TNM staging, complete clinical basic information, perioperative laboratory test results and postoperative follow-up data. The exclusion criteria included age younger than 20 years; pathology-confirmed non-hepatocellular cell carcinoma, preoperative chemotherapy and radiotherapy, preoperative infectious diseases, autoimmune diseases and other malignant tumors, loss of follow-up and lack of perioperative hematology records.

\section{Endpoints}

The primary endpoints of this study were disease-free survival (DFS) and overall survival (OS). The endpoint of this study was OS, which was defined as the period from the patient's date of surgery to the time of death or last followup. DFS was defined as the interval between the date of surgery and the date of tumor recurrence or December 31, 2018. Follow-up was continued until December 2018 
or until the patient died. Secondary endpoints included NLR, LMR and SII and compare grade of postoperative complications, length of stay (LOS), dosage of sufentanil use and times of patients requiring rescue analgesia in both groups.

\section{Anesthesia care}

No premedication was given before anesthesia induction. Routine monitoring, including electrocardiography, pulse oximetry, end-tidal carbon dioxide, invasive blood pressure monitoring, and a central venous catheter, was used for each patient. In the GEA group, the patients received epidural puncture at mid-thoracic levels (T7-T9), followed by insertion of an epidural catheter. The patients were tested with $3 \mathrm{~mL}$ of $2 \%$ lidocaine through the epidural catheter before anesthesia induction. Patients in the GEA group received $8 \mathrm{~mL}$ of $0.375-0.5 \%$ ropivacaine after the induction of GA, plus $4 \mathrm{~mL}$ of ropivacaine every $50 \mathrm{~min}$ until the end of surgery. At the end of the operation, the patients in the GEA group received a patient-controlled epidural analgesia (PCEA) pump $(0.1 \%$ ropivacaine and $0.5 \mu \mathrm{g} / \mathrm{mL}$ sufentanil, background $3 \mathrm{~mL} / \mathrm{h}$, bolus $4 \mathrm{~mL}$, lockout time $15 \mathrm{~min}$ ) for $48 \mathrm{~h}$.

In all patients, GA was induced with sufentanil, propofol, and rocuronium in all patients. The patients were then endotracheally intubated and GA maintained with $2.0-3.0 \%$ sevoflurane mixed with oxygen/air. Repeated injections of sufentanil and rocuronium were given as necessary during the operation. The patients in the GA group received a patient-controlled intravenous analgesia (PCIA) pump $(0.6 \mu \mathrm{g} / \mathrm{mL}$ sufentanil, background $3 \mathrm{~mL} / \mathrm{h}$, bolus $4 \mathrm{~mL}$, lockout time $15 \mathrm{~min}$ ) for $48 \mathrm{~h}$. The PCEA and PCIA pumps were removed on POD 2, and flurbiprofen at a dosage of $100 \mathrm{mg}$ was used for postoperative pain rescue per times in both groups.

We retrospectively collected the data from the database of the FUSCC clinical information system. The medical information, including the baseline demographic characteristics, medical history, primary diagnosis, operative details (procedure type, anesthesia time and estimated blood loss), anesthesia methods, pathology results (tumor encapsulation, size, number, and vascular invasion), grade of surgical complications and the LOS, was recorded and reviewed for each patient. The neutrophil, lymphocyte, monocyte, and platelet counts were recorded within 3 days before surgery (preop), on the first day after surgery (POD 1) and on the third day after surgery (POD 3). NLR, LMR and SII were calculated based from routine laboratory tests. SII was defined as follows: SII $=$ neutrophil $\times$ platelet $/$ lymphocyte. We performed 3 years' follow-up (every three months in the 1st and 2nd year and every 6 months in the 3 rd year) by medical record review and telephone contact.

\section{Statistical analysis}

The primary goal of this retrospective analysis was to compare the effect of different anesthesia methods on the OS and DFS rates of patients with hepatocellular carcinoma who underwent liver tumor resection. The Kaplan-Meier method was used to calculate OS and DFS. The secondary goal of this retrospective analysis was to compare the effects of GA and GEA on the patients' perioperative inflammatory indicators and inflammatory scores and compare Grade of postoperative complications, LOS, dosage of sufentanil use and times of patients requiring rescue analgesia in both groups.

Categorical data are expressed as $\mathrm{N}(\%)$, and the chisquare test was used for differential analysis; continuous data are expressed as the mean \pm standard deviation, and the $t$-test for two independent samples was used for differential analysis. The Mann-Whitney test was used for differential analysis. Cox proportional hazards regression models were used to compare risk factors between the different groups by using univariate models. A two-sided 5\% $\alpha$ level was used to determine the statistically significant variables in the model. Variables that were significant in univariate analysis were put into a multivariate model using the forward conditional method, which was used to fit a multivariate model.

To adjust the selection bias in this retrospective study, we conducted a propensity score matching analysis. The model contained 16 covariates: age, ASA, surgical procedure, anesthesia time, tumor encapsulation, tumor number, preoperative AFP, surgical methods, surgical type, tumor size, TNM staging, vascular invasion, coagulation function, liver cirrhosis, estimated blood loss, blood transfusion. The Greedy $5 \rightarrow 1$ digit match algorithm was used to match the baseline covariates, so that the two study groups (GEA or GA) would have similar propensity scores. SPSS 17.0 was used to analyse the results, $\mathrm{P}$ value $<0.05$ was considered statistically significant.

\section{Results}

A total of 801 patients undergoing selective curative resection for hepatocellular carcinoma were enrolled in 
this study. After applied the exclusion criteria, 415 patients remained in the GEA group, and 386 remained in the GA group. After propensity score matching analysis, there were 386 patients remained in the GEA group, and 386 remained in the GA group (Figure S1) The patients' demographics, including age, gender, ASA grade, operative details, pathological results and TNM staging, were similar between the groups (Table 1). The standardized differences for all covariates were $5.36 \%$ in the post-matching cohort, suggesting substantial reduction of bias between the two groups (Table 1).

\section{Primary endpoint}

In this study, the median follow-up time for all patients was 36.2 months (95\% CI, 35.7 to 36.6). The Kaplan-Meier survival curves for the GA and GEA groups are shown in Figure 1. The shapes and Cox model analysis of the curves over time suggest that the OS rate at 3 years after surgery was significantly shorter in the patients in the GA group than in the patients in the GEA group (54.2\% vs. $62.3 \%$, $\mathrm{P}<0.001$, Figure $1 A$ ), with a hazard ratio of $1.60(95 \%$ CI, 1.23 to $1.73 ; \mathrm{P}<0.001)$. The DFS rate at 3 years after surgery was also significantly lower in the patients in the GA group than in the patients in the GEA group $(41.2 \% v s$. $52.5 \%, \mathrm{P}<0.001$; Figure $1 B)$, with a hazard ratio of 1.34 (95\% CI, 1.07 to $1.60 ; \mathrm{P}<0.001)$.

The OS and DFS against anesthesia method and other variables were compared in a univariate Cox model and then in a multivariable Cox regression. In the univariate analysis, we observed that the anesthesia method, age, AFP level, vascular invasion and the ASA status were associated with a worse impact on OS and DFS (Table 2). GA was related with an HR (95\% CI) of 1.28 (1.07-2.02) for OS and an HR (95\% CI) of 1.06 (1.03-1.71) for DFS after multivariable analysis for known confounding factors (Table 3). Other variables related to a significant increase in the hazard of death after multivariable analysis included age, higher AFP level, vascular invasion, and higher ASA status. The multivariate analysis after propensity score matching

Table 1 Patient and treatment characteristics for both groups

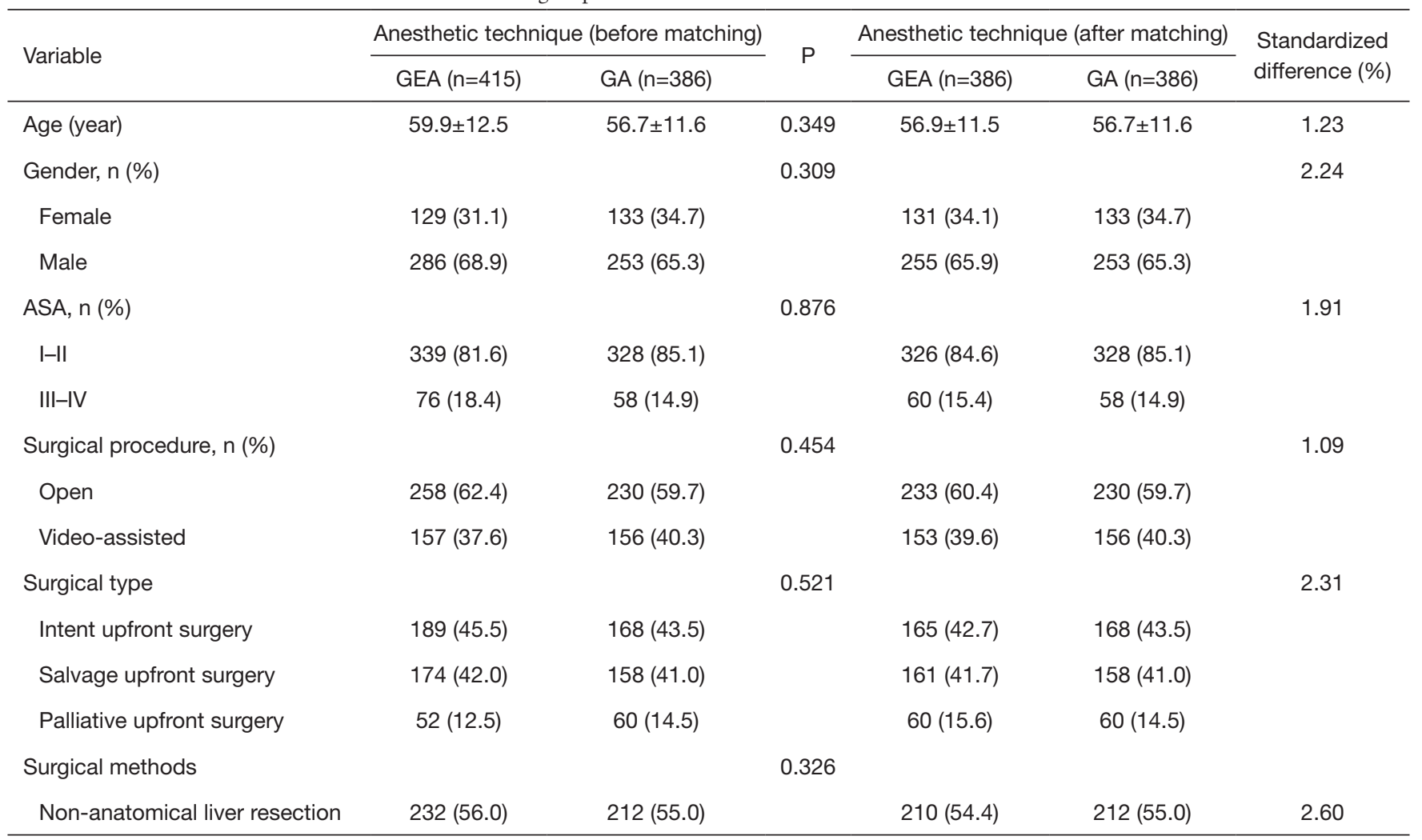

Table 1 (continued) 
Table 1 (continued)

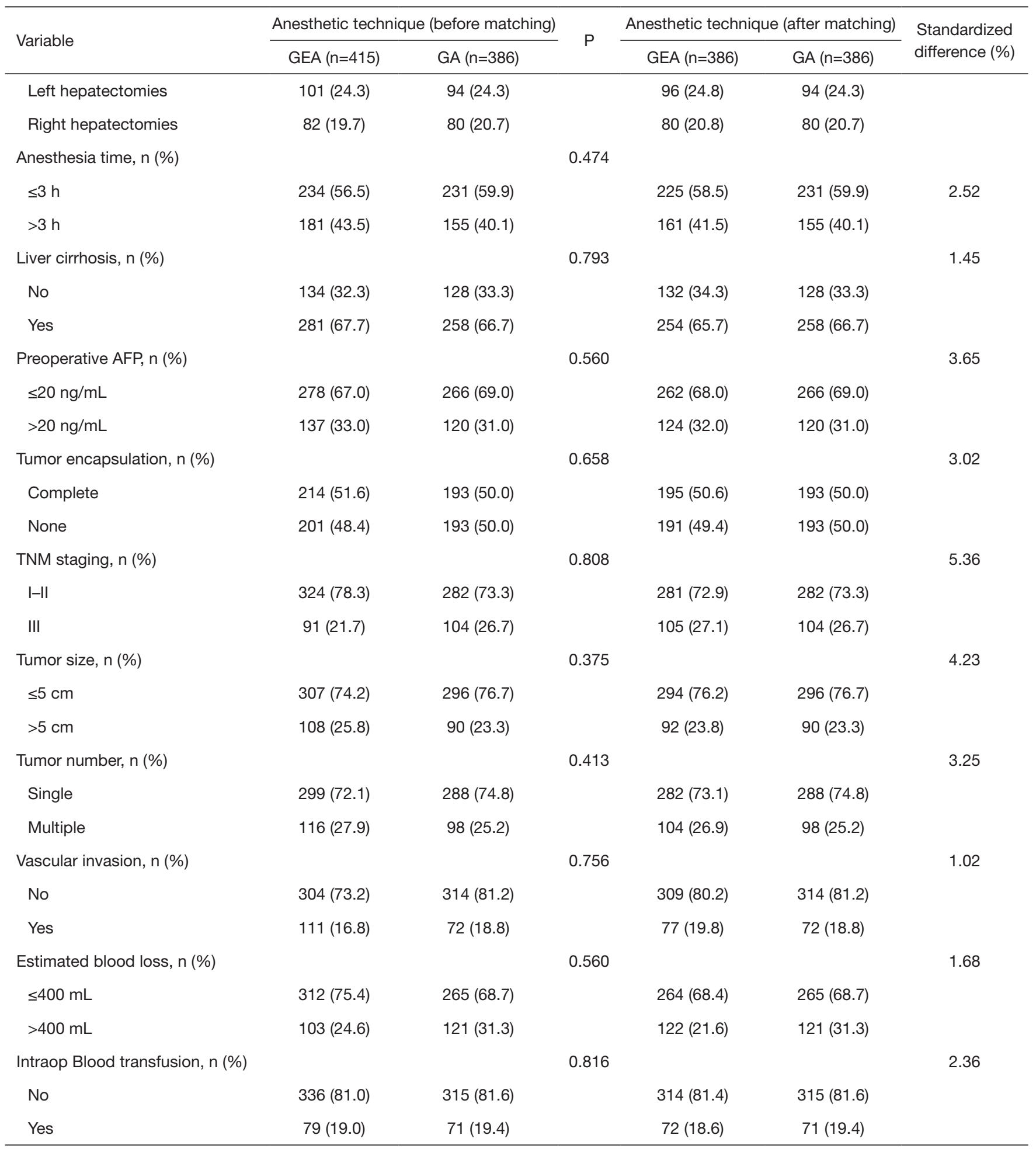

Data shown as mean \pm SD or $n(\%)$. Grade of surgical complications: Clavien-Dindo classification. GEA, general anesthesia combined with thoracic epidural anesthesia; GA, general anesthesia; AFP, alpha-fetoprotein; ASA, American Society of Anesthesiologists. P $\leq 0.05$ was considered statistically significant. 

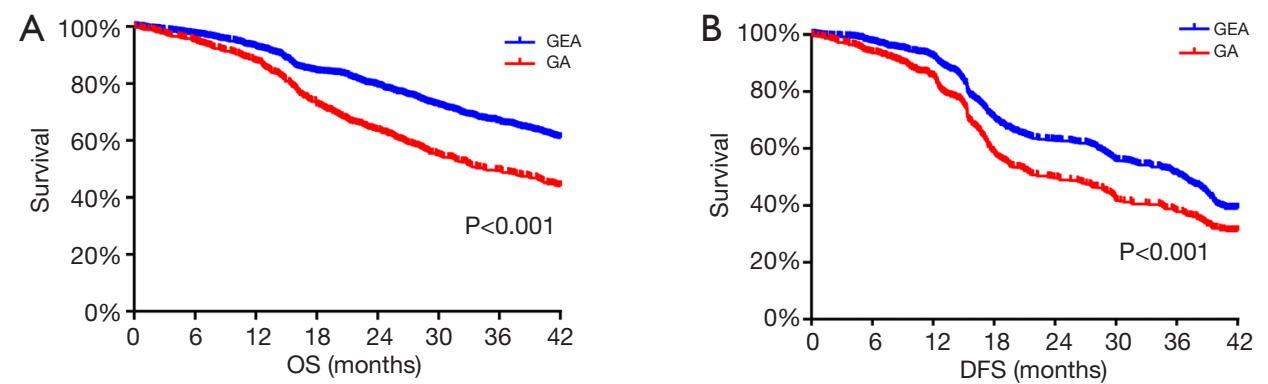

Figure 1 Impact of anesthesia methods on long-term outcomes in patients undergoing surgery for hepatocellular carcinoma. (A) OS curves from the date of surgery by anesthesia method; (B) DFS curves from the date of surgery by anesthesia method. OS, overall survival; DFS, disease-free survival; GEA, general-epidural anesthesia; GA, general anesthesia.

Table 2 Univariate analysis of OS and DFS

\begin{tabular}{|c|c|c|c|c|}
\hline Variables & \multicolumn{2}{|c|}{ os } & \multicolumn{2}{|c|}{ DFS } \\
\hline Anesthesia (general anesthesia) & $1.60(1.23,1.73)$ & $<0.001$ & $1.34(1.07,1.60)$ & $<0.001$ \\
\hline Age (years) & $1.07(1.01,1.14)$ & 0.030 & $1.10(1.03,1.18)$ & 0.010 \\
\hline Gender (male) & $0.63(0.08,5.15)$ & 0.665 & $0.92(0.53,1.59)$ & 0.764 \\
\hline AFP (>20 ng/mL) & $1.69(1.13,2.53)$ & 0.011 & $1.44(1.04,1.98)$ & 0.026 \\
\hline Tumor encapsulation (none) & $2.36(1.02,5.42)$ & 0.044 & $1.55(0.90,2.67)$ & 0.115 \\
\hline Tumor staging (III-IV) & $1.80(0.96,3.35)$ & 0.066 & $1.47(1.06,2.04)$ & 0.022 \\
\hline Tumor size $(>5 \mathrm{~cm})$ & $1.39(0.72,2.70)$ & 0.323 & $1.20(0.78,1.83)$ & 0.410 \\
\hline ASA (III-IV) & $1.89(1.14,3.14)$ & 0.013 & $2.77(1.17,6.54)$ & 0.021 \\
\hline Surgical procedure (video-assisted) & $1.10(0.41,2.97)$ & 0.845 & $1.34(0.52,3.41)$ & 0.545 \\
\hline Blood loss (>400 mL) & $1.35(0.52,3.52)$ & 0.538 & $1.09(0.63,1.90)$ & 0.751 \\
\hline Anesthesia time (>3 h) & $1.39(0.66,2.93)$ & 0.395 & $1.34(0.59,3.04)$ & 0.482 \\
\hline
\end{tabular}

AFP, alpha-fetoprotein; ASA, American Society of Anesthesiologists; OS, overall survival; DFS, disease-free survival. P $\leq 0.05$ was considered statistically significant.

analysis demonstrated that GA were independent predictors of poor survival and high risk of recurrence (HR 1.28, 95\% CI: $1.07-2.02, \mathrm{P}<0.001$; HR 1.06, 95\% CI: $1.03-1.71$, $\mathrm{P}<0.001$ ), respectively (Table 3).

\section{Secondary outcomes}

There was no statistically significant difference in preoperative inflammatory indicators [systemic inflammation-based scores (NLR, LMR and SII)] between the two groups $(\mathrm{P}=0.331, \mathrm{P}=0.743, \mathrm{P}=0.252$; Figure $2 A, B, C$, respectively). The NLR and SII were significantly increased in all patients on the first day after the operation compared with before the operation $(\mathrm{P}<0.001$; Figure $2 A, C$, respectively), and they were slightly decreased on the third day after the operation compared with the first day after the operation (POD1) but still higher than before the operation $(\mathrm{P}<0.05$, respectively, Figure $2 A, C)$. The 
Table 3 Multivariable Cox proportional of OS and DFS

\begin{tabular}{|c|c|c|c|c|}
\hline Variables & \multicolumn{2}{|c|}{ OS } & \multicolumn{2}{|c|}{ DFS } \\
\hline Anesthesia (general anesthesia) & $1.28(1.07,2.02)$ & $<0.001$ & $1.06(1.03,1.71)$ & $<0.001$ \\
\hline Age (years) & $1.04(1.00,1.09)$ & 0.018 & $1.07(1.02,1.12)$ & 0.006 \\
\hline AFP (>20 ng/mL) & $1.57(1.08,2.11)$ & 0.022 & $1.25(0.84,1.85)$ & 0.267 \\
\hline Tumor staging (III-IV) & NA & & $1.12(0.76,1.66)$ & 0.576 \\
\hline Vascular invasion (yes) & $2.55(1.34,4.86)$ & 0.004 & $1.89(1.25,2.84)$ & 0.002 \\
\hline ASA (III-IV) & $1.75(1.04,2.56)$ & 0.039 & $1.34(1.04,1.69)$ & 0.045 \\
\hline
\end{tabular}

OS, overall survival; DFS, disease-free survival; AFP, alpha-fetoprotein; ASA, American Society of Anesthesiologists; NA, not applicable. $\mathrm{P} \leq 0.05$ was considered statistically significant.
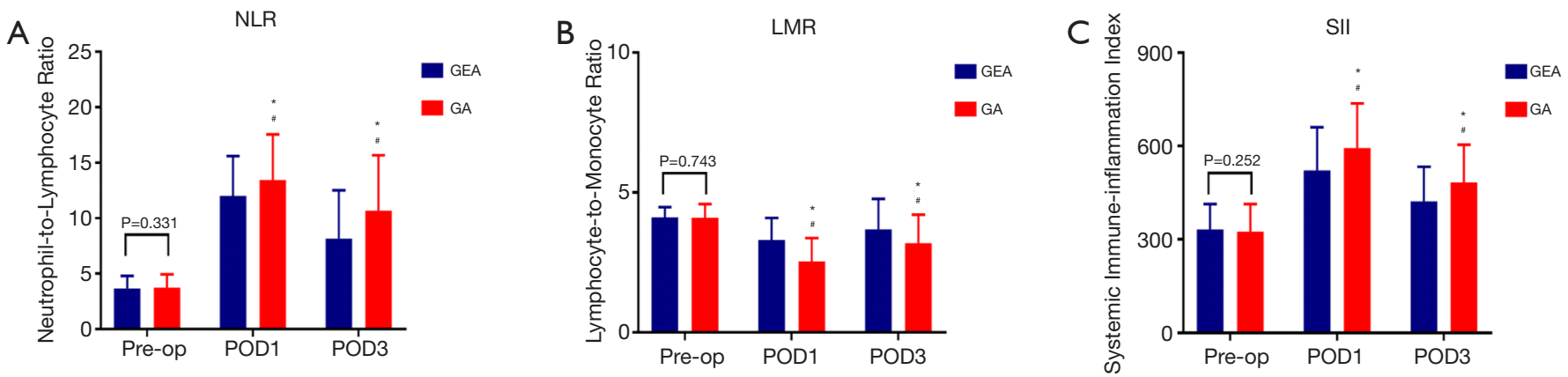

Figure 2 Perioperative NLR (A), LMR (B) and SII (C). $\left({ }^{*} \mathrm{P}<0.01\right.$ compared with GEA group; ${ }^{\text {P }}<0.01$ compared with pre-op). GEA, general-epidural anesthesia; GA, general anesthesia; NLR, neutrophil to lymphocyte ratio; LMR, lymphocyte to monocyte ratio; SII, system inflammatory index.

LMR was significantly decreased in all patients on the first day after the operation (POD1) compared with before the operation $(\mathrm{P}<0.001$, Figure $2 B)$. On the first and third days after the operation, both NLR and SII were significantly lower in the patients in the GEA group than in the patients in the GA group $(\mathrm{P}<0.001$, respectively, Figure $2 A, C)$, whereas LMR was significantly higher in the patients in the GEA group than in the patients in the GA group $(\mathrm{P}<0.001$, Figure $2 B$ ). Notably, the incidence of postoperative complications in the patients in the GEA group was lower $(\mathrm{P}<0.001$, Figure $3 A)$, and the average hospital stay of the patients in the GEA group was 7.4 days, which was less than that (10.8 days) of the patients in the GA group $(\mathrm{P}<0.001$, Figure $3 B)$. The average intraoperative sufentanil use and postoperative times of patients requiring rescue analgesia, were significantly lower in the GEA than in the GA group $(\mathrm{P}<0.001,31.6$ vs. 51.3, 1.6 vs. 4.6, respectively,
Figure $3 C, D)$.

\section{Discussion}

Surgery is one of the most important treatment methods for solid tumors, a series of studies have shown that surgery itself can promote the dissemination of circulating tumor cells, and surgical trauma can promote angiogenesis, further inhibit the already fragile anti-tumor immune function of cancer patients, and promote the dissemination and progression of micrometastases, thereby affecting the long-term prognosis of cancer patients $(5,16,17)$.

An increasing number of studies are paying close attention to the effects of anesthesia and other perioperative factors on the perioperative immune function and long-term prognosis of cancer patients (18). 

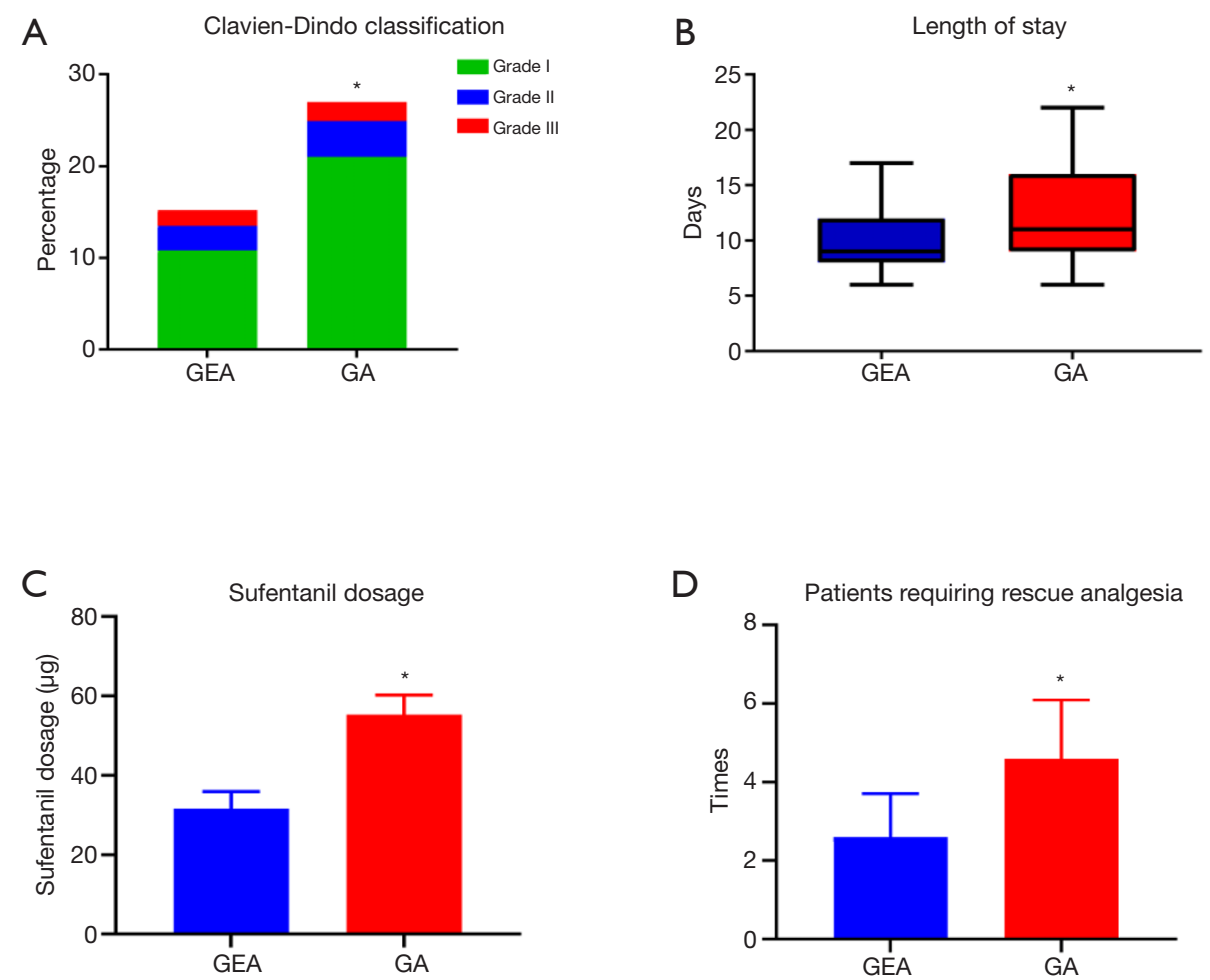

Figure 3 Impact of anesthesia methods on short-term outcomes in patients undergoing surgery for hepatocellular carcinoma. (A) Grade of postoperative complications, (B) LOS, (C) dosage of sufentanil use and (D) times of patients requiring rescue analgesia in both groups ( ${ }^{*} \mathrm{P}<0.01$ compared with GEA group). GEA, general-epidural anesthesia; GA, general anesthesia; LOS, length of stay.

Previous studies have shown that epidural anesthesia and postoperative analgesia can reduce the use of general anesthetics, especially opioids, during the operation, prevent harmful stimuli from being transmitted into the central nervous system, provide a better analgesic effect, and reduce excessive stress responses caused by surgical trauma, thereby better protecting the immune function of cancer patients $(8,19,20)$. Mounting evidence suggests that the systemic inflammatory response plays an important role in the pathogenesis and progression of tumors and is related to the OS of tumor patients $(21,22)$. Several indicators related to inflammatory response, such as NLR, LMR, and SII, have been noted for prognosis-related indicators in malignancies (23-25). However, the effects of epidural anesthesia and analgesia on perioperative systemic inflammatory indicators in patients undergoing liver cancer surgery are still unclear. The results of this study show that patients who received GEA had fewer fluctuations in perioperative systemic inflammatory response, indicating that epidural anesthesia and analgesia can better protect the anti-tumor immune function of patients undergoing selective curative resection for hepatocellular carcinoma. This result is also consistent with our previous findings in colorectal cancer patients (12).

Postoperative acute pain is the main cause of hypothalamic-pituitary-adrenal axis (HPA axis) activation, which can lead to decreased NK cell activity and Th cell imbalance as well as immunosuppression $(26,27)$. Opioids are one of the main drugs used for postoperative analgesia, but studies have shown that opioids can suppress antitumor immune function both directly and indirectly (28). The results of this study show that patients who received epidural anesthesia and analgesia used less opioids and had fewer instances of postoperative rescue analgesia, which indirectly suggests that patients who received epidural anesthesia and analgesia had an even better postoperative analgesic effect with the use of less opioids, which may also be the reason for the lower fluctuation in perioperative systemic inflammatory indicators in the patients of the GEA group.

For a long time, there has been controversy about the relationship between anesthesia methods and the long- 
term prognosis of cancer patients. Some studies have suggested that regional block anesthesia can improve the long-term prognosis of tumor patients $(29,30)$, while others have suggested that anesthesia methods are not associated with the long-term prognosis of this patient population (31). A meta-analysis suggested that the effect of the anesthesia method on the long-term prognosis of tumor patients may be associated with the type of tumor (9). The results of this study indicate that both the postoperative OS rate and the postoperative relapse-free survival rate of liver cancer patients who received epidural anesthesia and analgesia were higher than those of the patients who received GA alone. Multivariate analysis also showed that GA alone was an independent risk factor for postoperative survival in patients with liver cancer. For other anesthetic agents, lidocaine is a widely used amide local anesthetic that can reduce general anesthetic use, minimize opioid consumption, and provide adequate analgesia when given systemically during oncological surgery (32). In addition, lidocaine has shown promising anticancer properties. Different mechanisms have been described as being responsible for the antimetastatic effects of lidocaine, including TRPV6 receptor inhibition (33), reduced epidermal growth factor activity (34), and timeand dose-dependent deoxyribonucleic acid demethylation in different cancer cell lines (35).

Our study has several limitations. The study was retrospective and not randomized; therefore, the risk of confounding is high. The data were come from a single center. Liver cancer is often accompanied by coagulation abnormalities, which is a contraindication for epidural anesthesia; therefore, patients who receive GA alone may have poor coagulation, which could also have had an impact on the results of this study. This study did not analyse the influence of other factors, such as perioperative fluid therapy and body temperature, on the prognosis of tumor patients. Although epidural anesthesia and analgesia may protect the perioperative anti-tumor immune function of patients through a variety of mechanisms and has become an important part of the enhanced recovery after surgery (ERAS) strategy for various cancer surgeries, including liver cancer $(36,37)$, its effect on the mid- and long-term prognosis of tumor patients requires further clarification through multicenter prospective controlled studies.

\section{Conclusions}

In conclusion, this study suggests that epidural anesthesia and analgesia may reduce the impact of surgical trauma and stress on the perioperative systemic inflammatory response in patients undergoing scheduled liver cancer surgery, may protect the immune surveillance function after surgery and improve the long-term prognosis of patients with primary liver cancers. The results of this study need to be further validated by randomized controlled trials.

\section{Acknowledgments}

Funding: This research was supported by the National Natural Science Foundation of China (No. 81873948, 81871591), National Key R\&D Program of China (No. 2018YFC2001904), Shanghai Shenkang Hospital Development Center Clinical Science and Technology Innovation Project (No. SHDC12018105).

\section{Footnote}

Reporting Checklist: The authors have completed the STROBE reporting checklist. Available at http://dx.doi. org/10.21037/atm-20-3704

Data Sharing Statement: Available at http://dx.doi. org/10.21037/atm-20-3704

Conflicts of Interest: All authors have completed the ICMJE uniform disclosure form (available at http://dx.doi. org/10.21037/atm-20-3704). The authors have no conflicts of interest to declare.

Ethical Statement: The authors are accountable for all aspects of the work in ensuring that questions related to the accuracy or integrity of any part of the work are appropriately investigated and resolved. The study was conducted in accordance with the Declaration of Helsinki (as revised in 2013). This study was approved by the Ethics Committee of Fudan University Shanghai Cancer Centre (FUSCC), China (No. 202003011-3). All patients were consent the data for research use when receive treatment

Open Access Statement: This is an Open Access article distributed in accordance with the Creative Commons Attribution-NonCommercial-NoDerivs 4.0 International License (CC BY-NC-ND 4.0), which permits the noncommercial replication and distribution of the article with the strict proviso that no changes or edits are made and the original work is properly cited (including links to both the 
formal publication through the relevant DOI and the license). See: https://creativecommons.org/licenses/by-nc-nd/4.0/.

\section{References}

1. Bray F, Ferlay J, Soerjomataram I, et al. Global cancer statistics 2018: GLOBOCAN estimates of incidence and mortality worldwide for 36 cancers in 185 countries. CA Cancer J Clin 2018;68:394-424.

2. Chen $W$, Zheng R, Baade PD, et al. Cancer statistics in China, 2015. CA Cancer J Clin 2016;66:115-32.

3. Villanueva A. Hepatocellular Carcinoma. N Engl J Med 2019;380:1450-62.

4. Vogel A, Cervantes A, Chau I, et al. Hepatocellular carcinoma: ESMO Clinical Practice Guidelines for diagnosis, treatment and follow-up. Ann Oncol 2018;29:iv238-55.

5. Tohme S, Simmons RL, Tsung A. Surgery for Cancer: a Trigger for Metastases. Cancer Res 2017;77:1548-52.

6. Krall JA, Reinhardt F, Mercury OA, et al. The systemic response to surgery triggers the outgrowth of distant immune-controlled tumors in mouse models of dormancy. Sci Transl Med 2018;10:eaan3464.

7. Hiller JG, Perry NJ, Poulogiannis G, et al. Perioperative events influence cancer recurrence risk after surgery. Nat Rev Clin Oncol 2018;15:205-18.

8. Chen WK, Miao CH. The effect of anesthetic technique on survival in human cancers: a meta-analysis of retrospective and prospective studies. PLoS One 2013;8:e56540.

9. Weng $M$, Chen $W$, Hou $W$, et al. The effect of neuraxial anesthesia on cancer recurrence and survival after cancer surgery: an updated meta-analysis. Oncotarget 2016;7:15262-73.

10. Chen WK, Ren L, Wei Y, et al. General anesthesia combined with epidural anesthesia ameliorates the effect of fast-track surgery by mitigating immunosuppression and facilitating intestinal functional recovery in colon cancer patients. Int J Colorectal Dis 2015;30:475-81.

11. Xu YJ, Chen WK, Zhu Y, et al. Effect of thoracic epidural anaesthesia on serum vascular endothelial growth factor $\mathrm{C}$ and cytokines in patients undergoing anaesthesia and surgery for colon cancer. Br J Anaesth 2014;113 Suppl 1:i49-55.

12. Dolan RD, Lim J, McSorley ST, et al. The role of the systemic inflammatory response in predicting outcomes in patients with operable cancer: Systematic review and metaanalysis. Sci Rep 2017;7:16717.
13. Bilen MA, Martini DJ, Liu Y, et al. The prognostic and predictive impact of inflammatory biomarkers in patients who have advanced-stage cancer treated with immunotherapy. Cancer 2019;125:127-134.

14. Ní Eochagáin A, Burns D, Riedel B, et al. The effect of anaesthetic technique during primary breast cancer surgery on neutrophil-lymphocyte ratio, platelet-lymphocyte ratio and return to intended oncological therapy. Anaesthesia 2018;73:603-11.

15. Vicente D, Patino M, Marcus R, et al. Impact of epidural analgesia on the systemic biomarker response after hepatic resection Oncotarget 2019;10:584-594.

16. Li W, Zhou X, Huang Z, Zhang H, et al. Laparoscopic surgery minimizes the release of circulating tumor cells compared to open surgery for hepatocellular carcinoma. Surg Endosc 2015;29:3146-53.

17. Sullivan R, Alatise OI, Anderson BO, et al. Global cancer surgery: delivering safe, affordable, and timely cancer surgery. Lancet Oncol 2015;16:1193-224.

18. Horowitz M, Neeman E, Sharon E, et al. Exploiting the critical perioperative period to improve long-term cancer outcomes. Nat Rev Clin Oncol 2015;12:213-26.

19. Byrne K, Levins KJ, Buggy DJ. Can anesthetic-analgesic technique during primary cancer surgery affect recurrence or metastasis? Can J Anaesth 2016;63:184-92.

20. Wall T, Sherwin A, Ma D, et al. Influence of perioperative anaesthetic and analgesic interventions on oncological outcomes: a narrative review. Br J Anaesth 2019;123:135-50.

21. Gonzalez H, Hagerling C, Werb Z. Roles of the immune system in cancer: from tumor initiation to metastatic progression. Genes Dev 2018;32:1267-84.

22. Dupré A, Malik HZ. Inflammation and cancer: What a surgical oncologist should know. Eur J Surg Oncol 2018;44:566-70.

23. Wang Y, Peng C, Cheng Z, et al. The prognostic significance of preoperative neutrophil-lymphocyte ratio in patients with hepatocellular carcinoma receiving hepatectomy: a systematic review and meta-analysis. Int J Surg 2018;55:73-80.

24. Yang YT, Jiang JH, Yang HJ, et al. The lymphocyte-tomonocyte ratio is a superior predictor of overall survival compared to established biomarkers in HCC patients undergoing liver resection. Sci Rep 2018;8:2535.

25. Hu B, Yang XR, Xu Y, et al. Systemic immuneinflammation index predicts prognosis of patients after curative resection for hepatocellular carcinoma. Clin Cancer Res 2014;20:6212-22. 
26. Chapman CR, Tuckett RP, Song CW. Pain and stress in a systems perspective: reciprocal neural, endocrine, and immune interactions. J Pain 2008;9:122-45.

27. Borsook D, George E, Kussman B, et al. Anesthesia and perioperative stress: consequences on neural networks and postoperative behaviors. Prog Neurobiol 2010;92:601-12.

28. Al-Hashimi M, Scott SW, Thompson JP, et al. Opioids and immune modulation: more questions than answers. $\mathrm{Br}$ J Anaesth 2013;111:80-8.

29. Exadaktylos AK, Buggy DJ, Moriarty DC, et al. Can anesthetic technique for primary breast cancer surgery affect recurrence or metastasis? Anesthesiology 2006;105:660-4.

30. Zimmitti G, Soliz J, Aloia TA, et al. Positive Impact of Epidural Analgesia on Oncologic Outcomes in Patients Undergoing Resection of Colorectal Liver Metastases. Ann Surg Oncol 2016;23:1003-11.

31. Weingarten TN, Taccolini AM, Ahle ST, et al. Perioperative management and oncological outcomes following radical cystectomy for bladder cancer: a matched retrospective cohort study. Can J Anaesth 2016;63:584-95.
32. Dunn LK, Durieux ME. Perioperative Use of Intravenous Lidocaine. Anesthesiology 2017;126:729-37.

33. Piegeler T, Votta-Velis EG, Liu G, et al. Antimetastatic potential of amide-linked local anesthetics: inhibition of lung adenocarcinoma cell migration and inflammatory Src signaling independent of sodium channel blockade. Anesthesiology 2012;117:548-59.

34. Sakaguchi M, Kuroda Y, Hirose M. The antiproliferative effect of lidocaine on human tongue cancer cells with inhibition of the activity of epidermal growth factor receptor. Anesth Analg 2006;102:1103-7.

35. House CD, Vaske CJ, Schwartz AM, et al. Voltagegated $\mathrm{Na}+$ channel SCN5A is a key regulator of a gene transcriptional network that controls colon cancer invasion. Cancer Res 2010;70:6957-67.

36. Melloul E, Hubner M, Scott M, et al. Guidelines for Perioperative Care for Liver Surgery: enhanced Recovery After Surgery (ERAS) Society Recommendations. World J Surg 2016;40:2425-40.

37. Ljungqvist O, Scott M, Fearon KC: enhanced Recovery After Surgery: a Review. JAMA Surg 2017;152:292-8.

Cite this article as: Zhang $\mathrm{H}$, Guo K, Sun X, Liu Y, Zhong Z, Zhou W, Zeng W, Wang L, Cata JP, Chen W, Miao C. Impact of anesthesia methods on perioperative systemic inflammation and long-term outcomes in patients undergoing surgery for hepatocellular carcinoma: a propensity score-matched analysis. Ann Transl Med 2021;9(1):49. doi: 10.21037/atm-20-3704 